\title{
Liver disease in patients with diabetes mellitus
}

\author{
K. J. FoSTER \\ B.Sc., D.M., M.R.C.P. \\ A. H. GRIFFITH \\ K. Dewbury \\ B.Sc., M.B., F.R.C.R. \\ C. P. Price \\ B.Sc., Ph.D., M.C.B., M.R.I.C. \\ R. WRIGHT \\ M.A., M.D., D.Phil., F.R.C.P. \\ Departments of Medicine, Chemical Pathology and Radiology, Southampton University Hospitals
}

\begin{abstract}
Summary
Liver function tests were assessed in 60 unselected out-patient diabetics stabilized on insulin or oral hypoglycaemic agents. Routine liver function tests, particularly plasma concentrations of $\gamma$-glutamyl transpeptidase and alkaline phosphatase were elevated occasionally but rarely to more than twice the upper limit of normal. There was no correlation between measures of diabetic control and results of liver function tests. Twelve ( $20 \%)$ patients had evidence of gall stones, a prevalence above the expected from the community. Fourteen ( $23 \%)$ patients had an abnormally bright liver ultrasound echo pattern, probably indicative of fatty infiltration of the liver. This echo pattern was associated with only a minimal rise in plasma alanine amino transferase and alkaline phosphatase concentrations. It is concluded that functionally significant liver disease is uncommon amongst stabilized diabetic patients.
\end{abstract}

\section{Introduction}

Disturbance of liver function in some patients with diabetes mellitus is well recognized (Bradley, Sagild and Schertenleib, 1955; Camerini-Davalos, Marble and Muench, 1962) but the precise relationship of diabetes mellitus to liver dysfunction has not been simply established. The liver is probably the single most important regulatory site of glucose metabolism (Felig and Sherwin, 1976) and, in patients with liver disease, disturbance of carbohydrate metabolism is common (Johnston and Alberti, 1976). Various authors have reported a prevalence of cirrhosis in diabetics from 6 to $21 \%$

Correspondence: R. Wright, Professorial Medical Unit, Level F, Centre Block, Southampton General Hospital, Tremona Road, Southampton SO9 4XY at post-mortem (Creutzfeld, Frerichs and Sickinger, 1970), but it is likely that this figure is falsely elevated by inclusion of patients with hepatogenous diabetes whose prognosis may be very different from that of those with 'genetic' diabetes mellitus (Felig and Sherwin, 1976). Other studies have sought to exclude patients known to have liver disease (Camerini-Davalos et al., 1962).

This confusing situation has been compounded by the development and widespread application of new liver function tests since much of the previous work was completed. Moreover, it is difficult to avoid bias in selecting patients for study of liver function, particularly when biopsy is required to establish a pathological diagnosis. For these reasons the authors have sought to establish the frequency and severity of liver disease using modern techniques in an unselected population of established diabetics undergoing modern treatment. In particular, they have used grey scale ultrasound examination of the liver to derive information of the state of the liver parenchyma. They previously demonstrated a close correlation between the presence of an abnormally bright reflective echo pattern from the liver and either cirrhosis or fatty infiltration (Joseph, Dewbury and Maguire, 1979).

\section{Methods}

Sixty unselected diabetic patients requiring treatment with insulin or oral hypoglycaemic agents and attending a diabetic clinic were studied consecutively. Patients are usually referred to the clinic from local general practitioners for control of their diabetes and only a minority are referred for specific complications. Patients were interviewed and examined. A Vickers M300 autoanalyser was 
used routinely to measure plasma creatinine (by pseudo-kinetic Jaffé reaction), albumin (by a modified bromcresol green method), bilirubin (by diazoreaction with dichloraniline and Brij 35 as surfactant), aspartate and alanine amino transferase (by optimized methods using the respective ketoacid and tris buffer at $37^{\circ} \mathrm{C}$ ), and alkaline phosphatase (using nitrophenylphosphate as substrate). Plasma $\gamma$-glutamyl transpeptidase was measured by a kinetic method using $\gamma$-glutamyl nitro anilide as substrate with glycylglycine as acceptor. Plasma was used for random measurement of glucose by

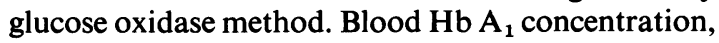
which is believed to reflect diabetic control (Peterson and Jones, 1977) was measured by the spectrophotometric method of Fluckiger and Winterhalter (1976), after storage for no more than 5 days as whole blood at $4^{\circ} \mathrm{C}$ with lithium heparin as anticoagulant. Preliminary studies showed fairly constant levels of $\mathrm{Hb} \mathrm{A}_{1}$ under these conditions after an initial rapid decay of about $6 \%$ of total $\mathrm{Hb} \mathrm{A}_{1}$. Twenty-four-hr collections of urine for urinary glucose excretion were also made. Ultrasound examinations of the liver and gall bladder were performed by an experienced operator (KD) in a standard manner using a Picker $80 \mathrm{~L}$ analogue B scanner with a focused $3 \mathrm{mHz}$ transducer. Examples of liver echo pattern regarded as normal or bright are shown in Figs 1 and 2 respectively. It was impractical to undertake ultrasound examination on a normal population. A retrospective survey was therefore made of the frequency of a bright echo pattern in 60 non-diabetic patients referred for ultrasonography for suspected gall stones which were not detected. These control subjects were matched for age and sex with the diabetic patients.

Weight was expressed as percent. ideal body weight (IBW) using the midpoint of the reference range for subjects of medium frame in the Metropolitan Life Insurance Co. Tables (Anonymous, 1964). For the purposes of assessment of alcohol intake, one 'drink' was taken to be half a pint of beer, one glass of wine or one measure of spirits. Results are expressed as mean \pm s.e. mean. Significance tests were made using Student's ' $t$ ' test and $\chi^{2}$ test. Linear correlations were sought between variables. Logarithmic transformation of grossly positively skewed data was used where appropriate.

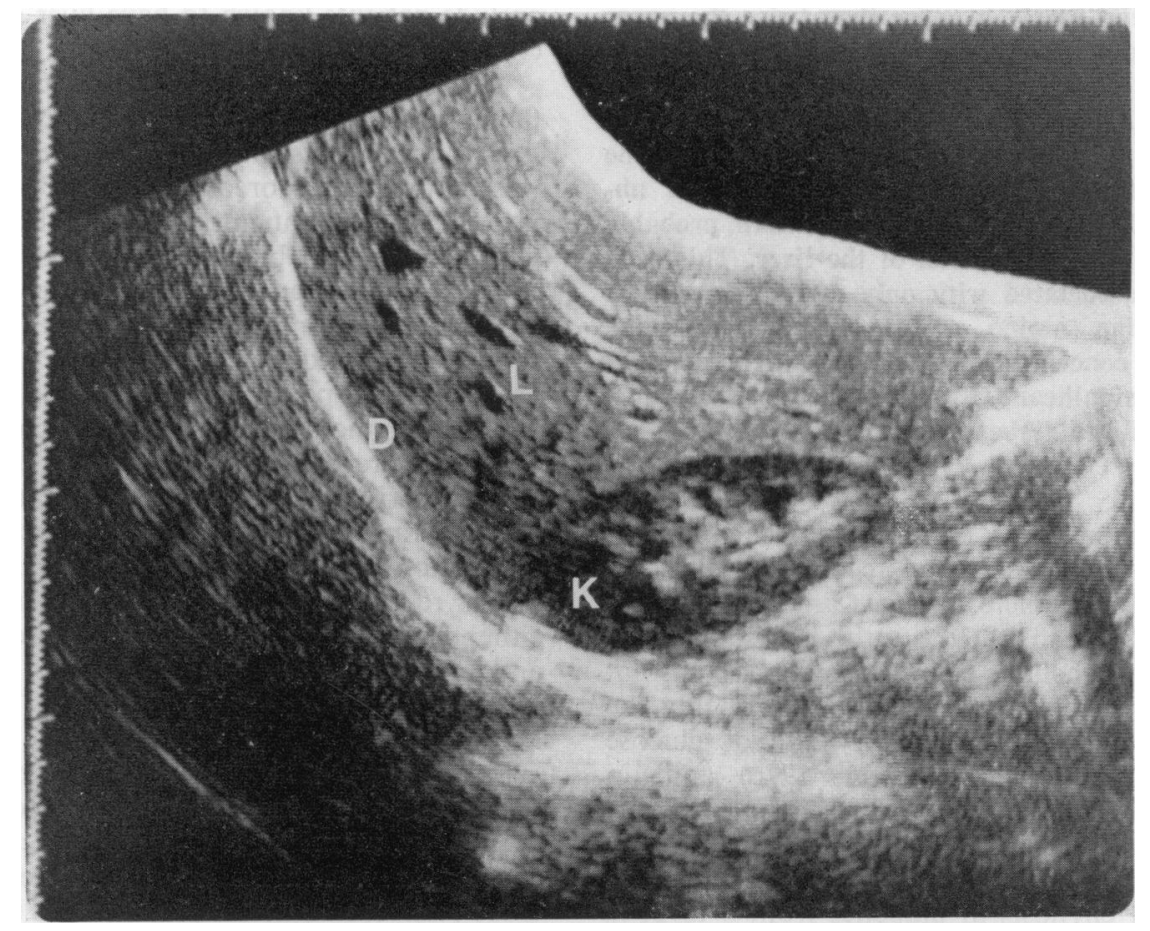

FIG. 1. Longitudinal scan through the right lobe of the liver showing the normal appearance. Note that the normal liver parenchyma (L) is interspersed with portal and hepatic veins, the walls of which can be identified. The kidney (K) is slightly less reflective than the liver. The diaphragm (D) stands out brightly in comparison with the liver. 


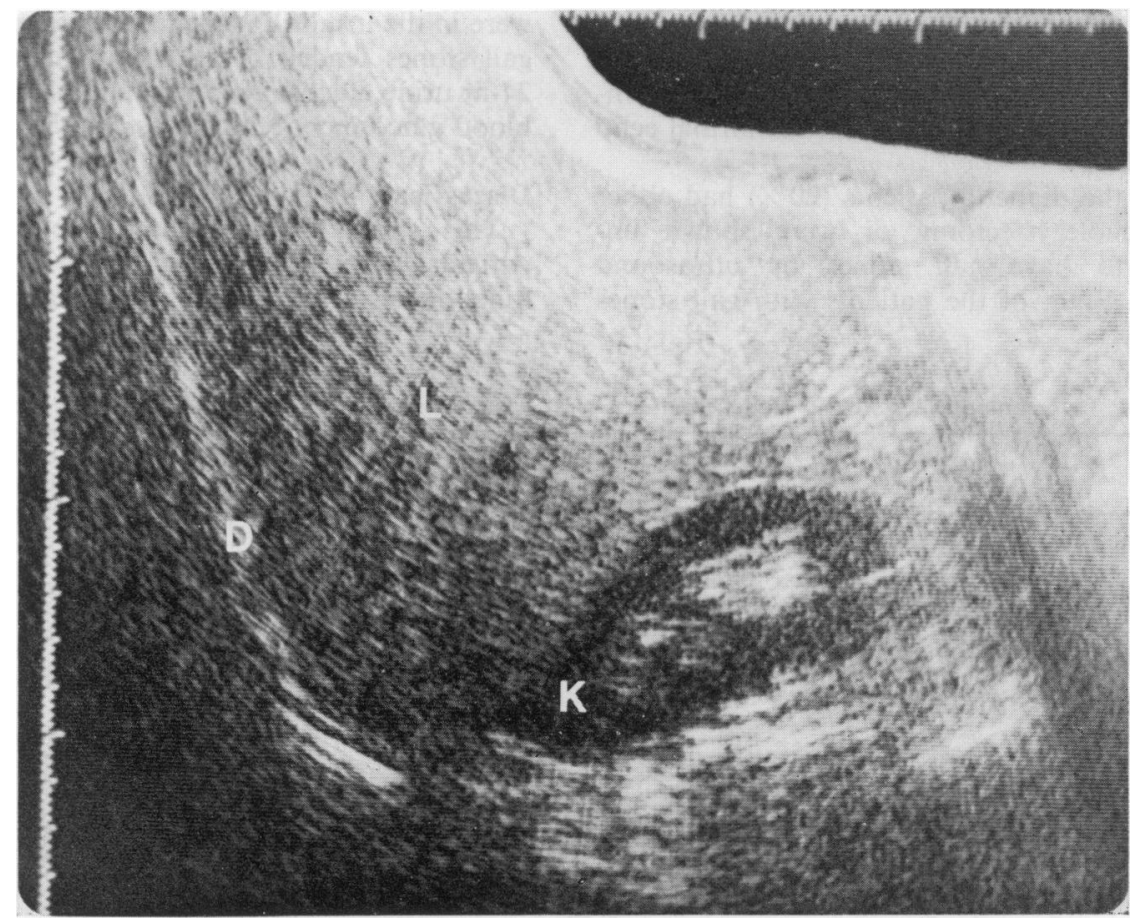

FIG. 2. Longitudinal scan through a brightly reflective liver (L). The overall amplitude of the echoes is higher than normal, the diaphragm (D) no longer appears so bright and the kidney (K) appears darker. The liver has a uniform appearance with loss of visualization of vessel walls.

\section{Patients}

Thirty-four insulin-dependent (18 male, 16 female) and 26 insulin-independent patients (19 male, 7 female) were studied. None had symptoms of uncontrolled diabetes mellitus. The mean age was $53 \pm$ 2 years (range 17 to 73 years), mean duration of diabetes $11 \pm 2$ years, (range 0.3 to 36 years) and mean weight $116 \pm 2 \%$ IBW (range $77-170 \%$ IBW). Insulin-dependent diabetics were younger but no less obese than the insulin-independent patients. Seven patients gave a history of previous viral hepatitis and 3 had undergone cholecystectomy. The patients estimated their current alcohol intake at $4 \pm 1$ drinks week (range 0 to 28 drinks) with a previous maximum intake of $11 \pm 3$ drinks/week (range 0 to 40 drinks/week). Twelve patients had retinopathy, 12 had symptoms of ischaemic heart or peripheral vascular disease and 14 patients had evidence of either peripheral or autonomic neuropathy. Only one patient was known to have nephropathy and mean plasma creatinine was $91 \pm 3 \mu \mathrm{mol} / 1$ (range 35-171 $\mu \mathrm{mol} / 1$ ).

\section{Results}

In Table 1 results of liver function tests in the diabetic patients are compared with normal reference ranges established for the methods used in this laboratory. Random blood glucose was increased, and $\mathrm{Hb} \mathrm{A}_{1}$ was significantly higher in the diabetics than in 15 healthy subjects $(7 \cdot 6 \% \pm$ s.e. mean $0 \cdot 3$, $P<0.01)$. Few patients had liver function test results consistently outside the reference range. There were no significant correlations between random blood glucose, 24-hr urinary glucose excretion, $\mathrm{Hb} \mathrm{A_{1 }}$ weight or alcohol consumption and liver function tests.

Fourteen $(23 \%)$ of the diabetics had a bright liver echo pattern, but only one of the control subjects had this echo pattern $\left(\chi^{2}=11.0, P<0.01\right)$. The control subject with a bright echo pattern had clinical, biochemical and histological evidence of alcoholic hepatitis. Fifteen of the 60 control subjects proved to have peptic ulcers, 6 to have cardiovascular disease, 4 irritable bowel syndrome, 13 had a variety of different conditions and in 20 patients the symptoms resolved spontaneously before a diagnosis was established.

Diabetics with a brightly reflective liver echo were heavier and had higher alanine amino transferase and alkaline phosphatase activity than those without 
a bright liver echo (Table 2). Seven of the 14 patients with a bright echo pattern had a liver edge palpable more than one $\mathrm{cm}$ below the costal margin compared with 12 of the 46 patients with a normal echo pattern $(P<0.05)$.

Twelve of the diabetic patients $(20 \%)$ had either undergone cholecystectomy or were shown unequivocally to have gall stones by ultrasound examination. Eight of the patients with gall stones were in the insulin-independent group. Patients with gall stones tended to be older and to have lower 24-hr urine glucose excretion and had lower random blood glucose concentrations (Table 2).

\section{Discussion}

This study confirms that a few diabetic outpatients have mild disturbance of routine liver function tests. It should be emphasized that these

TABLE 1. Liver function tests in 60 diabetic patients with reference ranges for methods used in this laboratory

\begin{tabular}{|c|c|c|c|}
\hline & $\begin{array}{c}\text { Laboratory reference } \\
\text { range }\end{array}$ & Diabetic patients* & $\begin{array}{l}\text { No. of diabetic patients } \\
\text { outside reference range }\end{array}$ \\
\hline Weight ( $\%$ ideal body weight) & - & $116 \pm 2$ & \\
\hline Random plasma glucose $(\mathrm{mmol} / \mathrm{l})$ & $3 \cdot 0-8 \cdot 6$ & $9 \cdot 9 \pm 0 \cdot 7$ & 27 \\
\hline 24-hr urine glucose (mmol/24 hr) & - & $43 \pm 11$ & \\
\hline $\mathrm{HbA}_{1}(\%$ total $\mathrm{Hb})$ & - & $11 \cdot 3 \pm 0 \cdot 4$ & \\
\hline Bilirubin $(\mu \mathrm{mol} / \mathrm{l})$ & $0-17$ & $\begin{array}{l}11 \pm 1 \\
(1-46)\end{array}$ & 6 \\
\hline Albumin $(g / l)$ & $32-50$ & $\begin{array}{l}41 \cdot 7 \pm 0 \cdot 7 \\
(38 \cdot 1-47 \cdot 1)\end{array}$ & 0 \\
\hline Alanine amino transferase (i.u./l) & $5-37$ & $\begin{array}{l}25 \pm 2 \\
(10-67)\end{array}$ & 6 \\
\hline Aspartate amino transferase (i.u./l) & $5-42$ & $\begin{array}{l}23 \pm 2 \\
(11-100)\end{array}$ & 2 \\
\hline Alkaline phosphatase (i.u./l) & $50-275$ & $\begin{array}{l}216 \pm 19 \\
(91-415)\end{array}$ & 11 \\
\hline$\gamma$-glutamyl transpepidase (i.u./l) & $\begin{array}{c}6-29(\mathrm{f}) \\
10-46(\mathrm{~m})\end{array}$ & $\begin{array}{c}27 \pm 2 \\
(8-83)\end{array}$ & 10 \\
\hline
\end{tabular}

* Figures in parenthesis indicate ranges; (f) female; (m) male.

TABLE 2. Characteristics of diabetic patients with and without abnormalities on liver ultrasound

\begin{tabular}{|c|c|c|c|c|}
\hline & \multicolumn{2}{|c|}{ Gall stones } & \multicolumn{2}{|c|}{ 'Bright echo' } \\
\hline & Patients without & Patients with & Patients without & Patients with \\
\hline Number & 48 & $12(20 \%)$ & 46 & $14(23 \%)$ \\
\hline Age (years) & $51 \pm 2$ & $59 \pm 3$ & $52 \pm 2$ & $56 \pm 2$ \\
\hline Weight ( $\%$ ideal body weight) & $116 \pm 2$ & $118 \pm 8$ & $114 \pm 2$ & $126 \pm 6^{*}$ \\
\hline Alcohol intake (drinks/week) & $4 \pm 2$ & $2 \pm 1$ & $3 \pm 2$ & $3 \pm 2$ \\
\hline Palpable hepatomegaly & 18 & $\frac{1}{1}$ & 12 & $\frac{1}{7}$ \\
\hline Creatinine $(\mu \mathrm{mol} / 1)$ & $92 \pm 3$ & $88 \pm 10$ & $96 \pm 9$ & $98 \pm 5$ \\
\hline Random plasma glucose $(\mathrm{nmol} / \mathrm{l})$ & $10 \cdot 6 \pm 0.8$ & $6 \cdot 9 \pm 0.8^{*}$ & $8 \cdot 8 \pm 0.5$ & $10.6 \pm 0.6$ \\
\hline 24 -hr urine glucose $(\mathrm{mmol} / \mathrm{l})$ & $50 \pm 12$ & $8 \pm 3$ & $38 \pm 11$ & $61 \pm 25$ \\
\hline Haemoglobin $A_{1}(\%)$ & $11 \cdot 1 \pm 0 \cdot 5$ & $11 \cdot 9 \pm 1 \cdot 0$ & $11 \cdot 2 \pm 0 \cdot 5$ & $11 \cdot 4 \pm 1 \cdot 1$ \\
\hline Bilirubin $(\mu \mathrm{mol} / \mathrm{l})$ & $12 \pm 1$ & $9 \pm 1$ & $11 \pm 1$ & $12 \pm 1$ \\
\hline Albumin $(g / l)$ & $42 \cdot 3 \pm 0 \cdot 3$ & $42 \cdot 5 \pm 0 \cdot 5$ & $42 \cdot 0 \pm 0 \cdot 3$ & $43 \cdot 3 \pm 0 \cdot 5$ \\
\hline Aspartate amino transferase (i.u./1) & $23 \pm 2$ & $23 \pm 2$ & $24 \pm 2$ & $22 \pm 2$ \\
\hline Alanine amino transferase (i.u./l) & $24 \pm 2$ & $26 \pm 6$ & $23 \pm 2$ & $32 \pm 4^{*}$ \\
\hline Alkaline phosphatase (i.u./l) & $212 \pm 73$ & $218 \pm 18$ & $203 \pm 8$ & $250 \pm 27^{*}$ \\
\hline$\gamma$-glutamyl transpeptidase (i.u./l) & $26 \pm 2$ & $33 \pm 7$ & $26 \pm 2$ & $35 \pm 2$ \\
\hline
\end{tabular}

* Significant difference, $P<0.05$. 
abnormalities were mild, rarely above twice the accepted upper limit of normal. As noted by others (Belfiore, Napoli and Lo Vecchio, 1973; Rosalki, 1975), elevation of plasma concentrations of alkaline phosphatase and $\gamma$-glutamyl transpeptidase were the abnormalities most often encountered, but these abnormalities remained minor and did not correlate with changes in other measures of liver function or measures of diabetic control. It seems unlikely that these minor abnormalities reflect clinically significant liver dysfunction in all cases. Coincidentally, none of the patients was known to have liver disease before the study and it would appear that such disease is uncommon in unselected diabetic patients. The authors suggest that the finding of more than mild disturbance of routine liver function in diabetic patients should prompt investigation just as in nondiabetic patients. Disturbance of metabolic functions of the liver appear to correlate with routine liver function tests (Johnston and Alberti, 1976) and thus impairment of the metabolic capacity of the liver is unlikely to contribute significantly to poor control of diabetes in the majority of diabetics.

None of the patients reported in this study had symptoms of uncontrolled diabetes although in some cases control was not satisfactory. The results may well differ from those in patients with uncontrolled diabetes. In a preliminary study of uncontrolled diabetic patients admitted to hospital the authors have noted more frequent disturbance of liver function tests, notably elevation of plasma alkaline phosphatase and $\gamma$-glutamyl transpeptidase concentrations and depression of plasma albumin. The latter probably reflects the cachexic effects of uncontrolled diabetes mellitus.

The authors used ultrasonography both to investigate the prevalence of gall stones and to assess the state of the liver parenchyma. This technique is particularly suited to screening large numbers of patients without bias imposed by fear of possible side effects. The accuracy of the technique in experienced hands in detecting gall stones is well recognized (Crade, 1979; Bastrum, Crow and Foote, 1977). For logistic reasons it was not possible to submit matched healthy control subjects to ultrasound examination but the observed overall prevalence of gall stones of $20 \%$ and the prevalence of $33 \%$ in the small number of maturity onset diabetics studied are undoubtedly higher than expected. In a careful study covering 9 towns in England, Barker and his colleagues (1979) found prevalences of $6 \%$ for women aged $<45$ years rising to $25 \%$ in those $>75$ years and of $4 \%$ and $17 \%$ in men of the same age ranges. The present study confirms evidence previously only available from post-mortem studies (Heaton, 1973). Creutzfeld and his co-workers (1970) suggested that the increased prevalence of gal 1 stones in diabetics might be due to obesity but this was not evident in the present patients, although all the authors' patients receive dietary advice to achieve their IBW. Surprisingly, gall stones were possibly associated with better control and it is of interest that bile saturation has been reported decreased in uncontrolled diabetes (Bennion and Grundy, 1977).

The frequency of a bright echo pattern in normal subjects is not known but is almost certainly very low. In contrast, approximately $90 \%$ of patients with either micronodular cirrhosis or moderate to severe fatty infiltration have bright echo patterns (Dewbury and Clark, 1979; Foster et al., 1980). Only one of the control group had a bright echo pattern despite bias imposed by using a group selected for investigation of the biliary tract, and that subject subsequently proved to have alcoholic liver disease. Previously the authors examined a group of $\mathbf{4 0}$ patients selected by finding normal liver histology on biopsy. Despite the bias arising from a group selected for investigation of the liver, only 2 had a bright echo pattern (Foster et al., 1980). In both groups the rate was significantly lower than the $23 \%$ rate observed in out-patient diabetics. The authors believe that in the majority of cases the bright echo pattern indicated fatty infiltration because of the lack of evidence of disturbed liver function, splenomegaly or clinical signs of chronic liver disease and because of the correlation with obesity. In no case did they think liver biopsy was justified on clinical grounds. Thus, the findings on liver ultrasound do not contradict the remarks made above on the basis of liver function tests about the frequency of functionally significant liver disease, although alanine amino transferase and alkaline phosphatase levels were marginally higher in the bright echo group. The prognostic significance of fatty infiltration of the liver is uncertain and they intend to follow the progress of these patients with a bright echo pattern.

\section{Acknowledgment} care.

Dr P. Todd kindly allowed us to study patients under his

\section{References}

ANONYMous (1964) Metropolital Life Insurance Co. Tables. In: Scienfific Tables, (Ed by Diem, K.). Documenta Geigy, Basle, Switzerland.

Barker, D.J.P., Gardner, M.J., Power, C. \& Hutt, M.S.R. (1979) Prevalence of gall stones at necropsy in nine British towns: a collaborative study British Medical Journal, 2, 1389.

Bartrum, R., Crow, H.C. \& Foote, S.R. (1977) Ultrasonic and radiographic cholecystography. New England Journal of Medicine, 296, 538.

Belfiore, F., Napoli, E. \& Lo Vecchio, L. (1973) Serum enzymes in diabetes mellitus. Clinical Chemistry, 19, 447. 
Bennion, L.J. \& Grundy, S.M. (1977) Effects of diabetes mellitus on cholesterol metabolism in man. New England Journal of Medicine, 296, 1365.

Bradley, R.F., Sagild, V. \& Schertenleib, F.E. (1955) Diabetes mellitus and liver function. New England Journal of Medicine, 253, 454.

Camerini-Davalos, R., Marble, A. \& Muench, H. (1962) Liver function in diabetes mellitus. New England Journal of Medicine, 266, 1349.

CRADE, M. (1979) Diagnostic Ultrasound in Gastrointestinal Disease, pp. 123-135. Churchill Livingstone, London, Edinburgh.

Creutzfeld, W., Frerichs, H. \& Sickinger, K. (1970) Liver disease and diabetes mellitus. Progress in Liver Diseases, 3, 371.

Dewbury, K.C. \& Clark, B. (1979) The accuracy of ultrasound in detection of cirrhosis of the liver. British Journal of Radiology, 52, 945.

Felig, P. \& Sherwin, R. (1976) Carbohydrate homoeostasis, liver and diabetes. Progress in Liver Diseases, 5, 149.
Fluckiger, R. \& Winterhalter, K.H. (1976) In vitro synthesis of haemoglobin Alc. Federation of European Biochemical Societies, 71, 356.

Foster, K.J., DeWbury, K.C., Griffith, A.H. \& Wright, R. (1980) The accuracy of ultrasound in detection of fatty infiltration of the liver. British Journal of Radiology, 53, 440.

Heaton, K.W. (1973) Epidemiology of gallstones and suggested etiology. Clinics in Gastroenterology, 2, 67.

Johnston, D.G. \& AlberTi, K.G.M.M. (1976) Carbohydrate metabolism in liver disease. Clinics in Endocrinology and Metabolism, 5, 675.

JosePh, A.E.A., Dewbury, K.C. \& McGuire, P.G. (1979) Ultrasound in the detection of chronic liver disease. British Journal of Radiology, 52, 184.

Peterson, C.M. \& Jones, R.L. (1977) Minor hemoglobins, diabetic control and diseases of postsynthetic protein modification. Annals of Internal Medicine, 87, 489.

RosalkI, S.B. (1975) Gamma-glutamyl transpeptidase. Advances in Clinical Chemistry, 17, 53. 